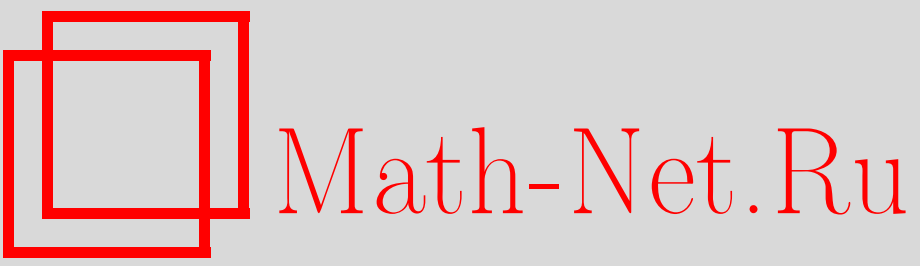

А. В. Анкилов, П. А. Вельмисов, Е. П. Семенова, О решениях интегродифференциальных уравнений в задаче динамики одной аэроупругой системы типа «тандем», Вестн. Сам. гос. техн. ун-та. Сер. Физ.-мат. науки, 2011, выпуск 2(), 266-271

DOI: https://doi.org/10.14498/vsgtu865

Использование Общероссийского математического портала Math-Net.Ru подразумевает, что вы прочитали и согласны с пользовательским соглашением http: //www. mathnet.ru/rus/agreement

Параметры загрузки:

IP: 3.85 .73 .92

26 апреля 2023 г., 14:53:28 


\title{
Механика
}

\author{
УДК 533.6.013.42
}

\section{О РЕШЕНИЯХ ИНТЕГРО-ДИФФЕРЕНЦИАЛЬНЫХ УРАВНЕНИЙ В ЗАДАЧЕ ДИНАМИКИ ОДНОЙ АЭРОУПРУГОЙ СИСТЕМЫ ТИПА «АНДЕМ»}

\author{
А.В. Анкилов, П. А. Велъмисов, Е. П. Семенова
}

Ульяновский государственный технический университет, 432027, Ульяновск, ул. Северный венец, 32.

E-mails: ankil@ulstu.ru, velmisov@ulstu.ru

Предложена математическая модель динамической системы двух упругих пластин типа «тандем», обтекаемых дозвуковым потоком газа (жидкости). Дано решение аэрогидродинамической части задачи, основанное на методах теории функиий комплексного переменного. Получена связанная система уравнений, позволяющая исследовать динамику пластин. Предложен численно-аналитический метод решения этих уравнений.

Ключевые слова: аэрогидроупругость, динамика, упругая пластина, система типа «тандем», деформачия, обтекание, дозвуковой поток.

Введение. При проектировании различных конструкций, устройств, приборов, аппаратов, систем и т. д., находящихся во взаимодействии с газожидкостной средой (обтекаемых потоком жидкости или газа), необходимо решать задачи, связанные с исследованием динамики и устойчивости упругих элементов, требуемой для их функционирования и надежности эксплуатации.

С одной стороны, воздействие потока может приводить к отрицательным эффектам, являющимся причиной нарушения необходимых функциональных свойств элементов вплоть до их разрушения (например, приводить к состоянию неустойчивости вследствие увеличения амплитуды или ускорения колебаний до критически допустимых значений). Такая проблема, когда неустойчивость является негативным явлением, возникает, например, при проектировании составных частей летательных и подводных аппаратов: элерона составной части крыла, руля высоты- составной части стабилизатора, руля направления - составной части киля, панели - составной части фюзеляжа, крыла или какой-либо другой части летательного аппарата.

В то же время для функционирования некоторых технических устройств явление возбуждения интенсивных колебаний при аэрогидродинамическом

Андрей Владимирович Анкилов (к.ф.-м.н., доц.), доцент, каф. высшей математики. Пётр Александрович Вельмисов (д.ф.-м.н., проф.), зав. кафедрой, каф. высшей математики. Елизавета Петровна Семенова, аспирант, каф. высшей математики. 
воздействии, указанное выше в качестве негативного, является необходимым. Примерами подобных устройств, относящихся к вибрационной технике и используемых для интенсификации технологических процессов, являются устройства для приготовления однородных смесей и эмульсий, в частности, устройства для подачи смазочно-охлаждающей жидкости в зону обработки.

В настоящей работе рассматривается модельная задача о динамике двух отдельно расположенных деформируемых пластин, решение которой является фундаментом для решения задач о динамике упругих элементов двух отдельно расположенных конструкций при обтекании их потоком газа или жидкости, например, о динамике элеронов крыльев двух летательных аппаратов, один из которых движется в следе другого.

Подобная задача об установившемся движении системы типа «тандем», составленной из двух плоских абсолютно жёстких пластинок, расположенных вдоль одной прямой, была рассмотрена в книге Л. И. Седова [1], в которой были вычислены гидродинамические силы, действующие на пластины. В настоящей работе, в отличие от [1], рассматривается система двух деформируемых пластин. При этом, в отличие от большого числа исследовавшихся (в том числе авторами этой статьи) ранее задач, пластины расположены отдельно друг от друга и не являются элементами одной и той же конструкции (например, элементами одного крылового профиля, элементами одной стенки канала и т. д.)

1. Постановка задачи. Рассматривается плоская задача аэрогидроупругости о малых колебаниях системы двух упругих пластин типа «тандем» (расположенных последовательно друг за другом вдоль одной линии) при дозвуковом обтекании их потоком идеальной несжимаемой среды (жидкости или газа). Пусть в состоянии покоя пластинам в физической плоскости $x O y$ соответствуют на оси $O x$ отрезки $\left[a_{1}, b_{1}\right]$ и $\left[a_{2}, b_{2}\right], a_{2}>b_{1}$. В бесконечно удаленной точке скорость газа равна $V$ и имеет направление, совпадающее с направлением оси $O x$. Предполагается, что прогибы пластин и возмущение однородного потока малы, то есть $\bar{w}_{k}(x, t)=\varepsilon w_{k}(x, t), \bar{\varphi}(x, y, t)=V x+$ $+\varepsilon \varphi(x, y, t), \varepsilon \ll 1, k=1,2$. Здесь $\bar{w}_{1}, \bar{w}_{2}$ и $\bar{\varphi}$ - соответственно прогибы и потенциал скорости возмущенного потока газа, а $w_{1}, w_{2}$ и $\varphi$-функции, определяющие прогибы (деформации) пластин и потенциал скорости; $x, y-$ декартовы координаты, $t$ - время.

Потенциал $\varphi$ удовлетворяет уравнению Лапласа

$$
\varphi_{x x}+\varphi_{y y}=0, \quad(x, y) \in G=\mathbb{R}^{2} \backslash\left(\left[a_{1}, b_{1}\right] \cup\left[a_{2}, b_{2}\right]\right),
$$

условию отсутствия возмущений в бесконечно удаленной точке

$$
\left(\varphi_{x}^{2}+\varphi_{y}^{2}+\varphi_{t}^{2}\right)_{\infty}=0
$$

и линеаризованным граничным условиям

$$
\varphi_{y}^{ \pm}=\dot{w}_{k}+V w_{k}^{\prime}, \quad x \in\left(a_{k}, b_{k}\right), \quad k=1,2,
$$


где $\varphi_{y}^{ \pm}=\lim _{y \rightarrow 0 \pm 0} \varphi_{y}(x, y, t)$. В формулах $(1)-(3)$ и далее точка обозначает частную производную по $t$, штрих - частную производную по $x$, индексы $x, y-$ частные производные по $x, y$.

Уравнения малых колебаний пластин в линейной постановке имеют вид $L_{k}\left(w_{k}\right)=\rho\left(\varphi_{t}^{+}-\varphi_{t}^{-}\right)+\rho V\left(\varphi_{x}^{+}-\varphi_{x}^{-}\right), x \in\left(a_{k}, b_{k}\right), y=0$, где $L_{k}\left(w_{k}\right)$ определяются выражениями

$$
L_{k}\left(w_{k}\right) \equiv M_{k} \ddot{w}_{k}+D_{k} w_{k}^{\prime \prime \prime \prime}+N_{k} w_{k}^{\prime \prime}+\delta_{k} \dot{w}_{k}^{\prime \prime \prime \prime}+\beta_{k} \dot{w}_{k}+\gamma_{k} w_{k},
$$

при этом $\rho, M_{k}, D_{k}, N_{k}, \delta_{k}, \beta_{k}, \gamma_{k}$ - некоторые постоянные, $k=1,2$.

2. Решение аэрогидродинамической части задачи. Выразим потенциал $\varphi(x, y, t)$ через функции прогибов $w_{k}(x, t)$. С этой целью в области $G$ введём комплексный потенциал $W=f(z, t)=\varphi+i \psi$, где $\psi=\psi(x, y, t)$ - функция тока, $z=x+i y$. Для функции скоростей $f_{z}(z, t)=\varphi_{x}-i \varphi_{y}$ согласно условиям (3) имеем следующее интегральное представление [1]:

$$
f_{z}(z, t)=\frac{1}{\pi \sqrt{h(z)}}\left(\int_{a_{2}}^{b_{2}} \frac{v_{2}(\tau, t)}{\tau-z} \sqrt{h(\tau)} d \tau-\int_{a_{1}}^{b_{1}} \frac{v_{1}(\tau, t)}{\tau-z} \sqrt{h(\tau)} d \tau+\Gamma(t)\right),
$$

где $h(z)=\left(z-a_{1}\right)\left(z-b_{1}\right)\left(z-a_{2}\right)\left(b_{2}-z\right) ; v_{k}(\tau, t)=\dot{w}_{k}(\tau, t)+V w_{k}^{\prime}(\tau, t)$; $\Gamma(t)$ - функция, определяющая циркуляцию скорости газа вокруг каждой пластины.

Используя методы теории функций комплексного переменного [2], получим связанную систему уравнений относительно функций прогибов $w_{1}(x, t)$ и $w_{2}(x, t)$, описывающую динамику двух упругих пластин в дозвуковом потоке газа [3]:

$$
\begin{gathered}
(-1)^{k} L_{k}\left(w_{k}\right)=\frac{2 \rho \sqrt{h(x)}}{\pi}\left(\int_{a_{1}}^{b_{1}} \frac{\tilde{v}_{1}(\tau, t)}{\sqrt{h(\tau)}} \frac{d \tau}{\tau-x}-\int_{a_{2}}^{b_{2}} \frac{\tilde{v}_{2}(\tau, t)}{\sqrt{h(\tau)}} \frac{d \tau}{\tau-x}\right)- \\
-\frac{2 \rho \Gamma^{\prime}(t)}{\pi} H_{k}(x)+\frac{2 \rho}{\pi} \int_{a_{1}}^{b_{1}} \frac{\tilde{v}_{1}(\tau, t)}{\sqrt{h(\tau)}} d \tau \int_{a_{k}}^{x} \frac{(\tau-\xi)\left(\tau+\xi-a_{0}\right)}{\sqrt{h(\xi)}} d \xi- \\
\quad-\frac{2 \rho}{\pi} \int_{a_{2}}^{b_{2}} \frac{\tilde{v}_{2}(\tau, t)}{\sqrt{h(\tau)}} d \tau \int_{a_{k}}^{x} \frac{(\tau-\xi)\left(\tau+\xi-a_{0}\right)}{\sqrt{h(\xi)}} d \xi+ \\
+\frac{2 \rho V}{\pi \sqrt{h(x)}}\left(\int_{a_{1}}^{b_{1}} \frac{v_{1}(\tau, t)}{\tau-x} \sqrt{h(\tau)} d \tau-\int_{a_{2}}^{b_{2}} \frac{v_{2}(\tau, t)}{\tau-x} \sqrt{h(\tau)} d \tau-\Gamma(t)\right)
\end{gathered}
$$

где $x \in\left(a_{k}, b_{k}\right), a_{0}=-\left(a_{1}+b_{1}+a_{2}+b_{2}\right) / 2$,

$$
\tilde{v}_{k}(\tau, t)=\int_{a_{k}}^{\tau}\left(\ddot{w}_{k}(x, t)+V \dot{w}_{k}^{\prime}(x, t)\right) d x, \quad H_{k}(x)=\int_{a_{k}}^{x} \frac{d \xi}{\sqrt{h(\xi)}},
$$

а функция $\Gamma(t)$ подобрана так, чтобы циркуляция вокруг каждой пластины равнялась нулю: 


$$
\begin{aligned}
\Gamma(t)=\frac{1}{H_{1}\left(b_{1}\right)}\left(\int_{a_{2}}^{b_{2}} \frac{d x}{\sqrt{h(x)}} \int_{a_{2}}^{b_{2}} \frac{v_{2}(\tau, t)}{x-\tau} \sqrt{h(\tau)} d \tau-\right. \\
\left.\quad-\int_{a_{1}}^{b_{1}} \frac{d x}{\sqrt{h(x)}} \int_{a_{1}}^{b_{1}} \frac{v_{1}(\tau, t)}{x-\tau} \sqrt{h(\tau)} d \tau\right) .
\end{aligned}
$$

3. Исследование динамики пластин. Предположим, что концы упругих пластин закреплены шарнирно (исследования для других закреплений проводятся аналогично). Проведём процедуру метода Галёркина для $m$ приближений:

$$
w_{n}(x, t)=\sum_{k=1}^{m} \omega_{k}^{(n)}(t) \sin \alpha_{k}^{(n)}\left(x-a_{n}\right), \quad \alpha_{k}^{(n)}=\pi k /\left(b_{n}-a_{n}\right), \quad n=1,2 .
$$

Записывая условия ортогональности невязок уравнений (5) к функциям $\left\{\sin \alpha_{i}^{(n)}\left(x-a_{n}\right)\right\}_{i=1}^{m}$, получим систему из $2 m$ обыкновенных дифференциальных уравнений для определения $\omega_{k}^{(n)}(t)$ :

$$
\sum_{n=1}^{2} \sum_{k=1}^{m}\left(A_{l i k}^{(n)} \ddot{\omega}_{k}^{(n)}(t)+B_{l i k}^{(n)} \dot{\omega}_{k}^{(n)}(t)+C_{l i k}^{(n)} \omega_{k}^{(n)}(t)\right)=0,
$$

где $A_{l i k}^{(n)}, B_{l i k}^{(n)}, C_{l i k}^{(n)}$ - постоянные коэффициенты, зависящие от параметров системы; $l=1,2$.

Для нахождения начальных значений $\omega_{k}^{(n)}(0), \dot{\omega}_{k}^{(n)}(0)$ воспользуемся начальными условиями $w_{n}(x, 0)=u_{n}(x), \dot{w}_{n}(x, 0)=v_{n}(x)$. Из условий ортогональности невязок начальных условий получим:

$$
\begin{aligned}
& \omega_{i}^{(n)}(0)=\frac{2}{b_{n}-a_{n}} \int_{a_{n}}^{b_{n}} u_{n}(x) \sin \alpha_{i}^{(n)}\left(x-a_{n}\right) d x, \\
& \dot{\omega}_{i}^{(n)}(0)=\frac{2}{b_{n}-a_{n}} \int_{a_{n}}^{b_{n}} v_{n}(x) \sin \alpha_{i}^{(n)}\left(x-a_{n}\right) d x .
\end{aligned}
$$

Таким образом, получена задача Коши для системы обыкновенных дифференциальных уравнений (7) с начальными условиями (8).

4. Численный эксперимент в задаче о динамике упругих пластин. На основе решения задачи Коши (7), (8) проведены численные эксперименты по исследованию динамики пластин при различных параметрах механической системы. Рассмотрим пример одной такой системы, состоящей из алюминиевых пластин, со следующими параметрами: $\rho=1$ - плотность рабочей среды (газ), $V=2$-скорость потока, $E=7 \cdot 10^{10}$ - модуль Юнга материала пластин, $\rho_{\text {пл }}=8480$ - плотность материала пластин, $a_{1}=0, b_{1}=1, a_{2}=2$, $b_{2}=4, h_{1}=h_{2}=h=0,005, M_{1}=M_{2}=\rho_{\text {пл }} h=42,4, \nu=0,31, D_{1}=D_{2}=$ $=E h^{3} /\left(12\left(1-\nu^{2}\right)\right)=806,7, N_{1}=N_{2}=-2000, \gamma_{1}=\gamma_{2}=4, \beta_{1}=\beta_{2}=0,1$, $\delta_{1}=\delta_{2}=0,2$. Здесь все значения приведены в международной системе измерения единиц СИ. 
Задача для модельной системы со следующими начальными условиями:

$$
\begin{array}{cl}
w_{1}(x, 0)=0,01 \cdot x^{3}(1-x)^{3}, & \dot{w}_{1}(x, 0)=-0,005 \cdot x^{4}(1-x)^{4}, \\
w_{2}(x, 0)=0,01 \cdot(x-2)^{3}(4-x)^{3}, & \dot{w}_{2}(x, 0)=-0,005 \cdot(x-2)^{4}(4-x)^{4}
\end{array}
$$

решалась с помощью системы Mathematica. На рисунке в качестве примера приведены графики функций $w_{1}\left(x_{1}, t\right), w_{2}\left(x_{2}, t\right)$, задаваемых соотношениями (6) при $m=4$, при $t \in[0 ; 1,5]$, где $x_{1}=0,5, x_{2}=3$.
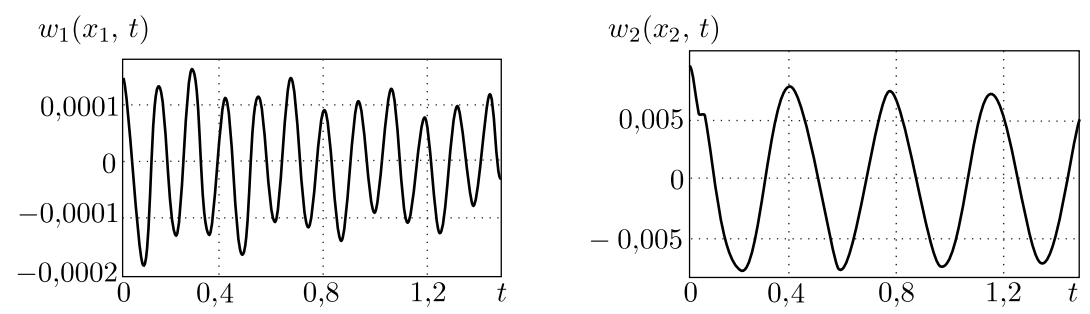

Деформация пластин в точках $x_{1}=0,5$ и $x_{2}=3$

Заключение. Предложена математическая модель динамической системы типа «тандем», состоящей из двух упругих пластин, последовательно расположенных друг за другом вдоль одной линии и обтекаемых дозвуковым потоком газа (жидкости). Дано решение аэрогидродинамической части задачи, основанное на методах теории функций комплексного переменного. На основе полученной связанной системы уравнений для прогибов пластин исследована их динамика.

Работа выполнена в рамках реализации ФЦП «Научные и научно-педагогические кадры инновационной России (2009-2013 гг.)» (гос. контракт № П1122).

\section{БИБЛИОГРАФИЧЕСКИЙ СПИСОК}

1. Седов Л. И. Плоские задачи гидродинамики и аэродинамики. М.: Наука, 1980. 448 с.; англ. пер.: Sedov L. I. Two-dimensional problems in hydrodynamics and aerodynamics. New York: John Wiley and Sons, 1965. 427 pp.

2. Лаврентъев М.А., Шабат Б. В. Методы теории функций комплексного переменного. M.: Наука, 1973. 736 c. [Lavrent'ev M. A., Shabat B. V. Methods in the theory of functions of complex variables. Moscow: Nauka, 1973. 736 pp.]

3. Анкилов А. В., Вельмисов П. А., Решетников Ю. А. Математическое моделирование одной динамической системы типа «тандем» // Журнал СВМO, 2010. Т. 12, № 3. С. 18-27. [Ankilov A.V., Vel'misov P. A., Reshetnikov Yu. A. A mathematical modeling of dynamical system "tandem" type// Zhurnal SVMO, 2010. Vol. 12, no.3. Pp. 18-27].

Поступила в редакцию 20/XII/2010;

в окончательном варианте - 25/III/2011. 
MSC: 35Q35

\section{ON SOLUTIONS OF INTEGRO-DIFFERENTIAL EQUATIONS IN DYNAMIC PROBLEM OF ONE AEROELASTIC SYSTEM "TANDEM" TYPE}

\section{A. V. Ankilov, P. A. Velmisov, E. P. Semenova}

Ulyanovsk State Technical University,

32, Severny venec st., Ulyanovsk, 432027, Russia.

E-mails: ankil@ulstu.ru, velmisov@ulstu.ru

A mathematical model of the dynamical system of the two elastic "tandem" type plates, flowing along of the subsonic flow of gas (fluid) is proposed. The solution of aerohydrodynamic part of problem based on the methods of complex variable functions theory is given. The bound system of the equations allowing to investigate the plates dynamic is obtained. The numerical-analytical method of solution of this equation is offered.

Key words: aerohydroelastisity, dynamic, elastic plate, "tandem" type system, deformation, flow, subsonic flow.

Original article submitted 20/XII/2010; revision submitted $25 / \mathrm{III} / 2011$.

Andrey V. Ankilov (Ph. D. (Phys. \& Math.)), Associate Professor, Dept. of Higher Mathematics. Petr A. Velmisov (Dr. Sci. (Phys. \& Math.)), Head of Dept., Dept. of Higher Mathematics. Elizaveta P. Semenova, Postgraduate Student, Dept. of Higher Mathematics. 